\title{
Detection of Chlamydia trachomatis antigen by enzyme immunoassay: importance of confirmatory testing
}

\author{
K Fonseca, D W Megran, C M Anand
}

\begin{abstract}
Aim-To determine when a fluorescence assay for Chlamydia trachomatis elementary bodies in the specimen buffer is of most value as a verification test for genital specimens reactive on screening enzyme immunoassay (EIA).

Method-Genital swabs from high and medium prevalence populations were tested using EIA. Samples with absorbance values greater than the positive threshold and those within the range of $30 \%$ below this value were verified by the MicroTrak direct fluorescence assay (DFA) test. Quotients derived from the threshold value and specimen absorbances were used to establish confidence limits for the EIA.

Results-Of 13283 swabs tested, 474 from the high risk group and 236 from the medium risk group were reactive on EIA and confirmed by DFA. Thirty six (5.9\%) patients with confirmed reactive samples would have been missed if the kit criteria alone were followed. When confidence limits were applied to the calculated quotients, only those samples with an EIA quotient of $\geq 4.0$ were universally confirmed by the DFA.

Conclusion-A scheme of testing which uses the DFA to verify EIA reactive specimens over a specified range was found to improve the sensitivity and specificity of the EIA screening test.

(F Clin Pathol 1995;48:214-217)
\end{abstract}

Provincial Laboratory of Public Health for Southern Alberta, 3030 Hospital Drive N.W., P.O. Box 2490, Calgary, Alberta, Canada T2P 2M7

$\mathrm{K}$ Fonseca

C M Anand

Department of Microbiology and Infectious Diseases, Faculty of Medicine, The University of Calgary, Calgary, Alberta, Canada

K Fonseca

D W Megran

Sexually Transmitted

Diseases Clinic

(Alberta Health),

Calgary, Alberta,

Canada

D W Megran

Correspondence to:

Dr K Fonseca.

Accepted for publication

4 August 1994
Keywords: Chlamydia trachomatis, enzyme immunoassay, antigen detection.

The use of the enzyme immunoassay (EIA) in screening for Chlamydia trachomatis in genital specimens is widely accepted. For most EIA kits, the signal strength or absorbance value is usually proportional to the amount of antigen present in the specimen; thus, a range of absorbance values may be encountered with each batch of specimens tested. The positive threshold provides a reference for assessing whether each sample's absorbance corresponds to a reactive result. This value is not absolute and incorporates statistical elements in its derivation, consequently both false positive and negative results are likely.

To improve the specificity of the EIA without sacrificing sensitivity, additional verification procedures for EIA reactive samples have employed blocking assays, ${ }^{12}$ preparation of an additional slide for direct immunofluorescence assay (DFA) testing, ${ }^{3}$ and DFA on the cytocentrifuged culture transport medium or EIA specimen buffer. ${ }^{45}$ In these studies the numbers of samples examined were small and/or the verification procedures were performed only on those found to be reactive or discrepant.

From previous evaluations of this and other kits (American Society of Microbiology Abstracts, 1989, and unpublished observations), we noted that when the DFA was performed on the sample buffer of specimens with absorbance values below the positive threshold, a number contained elementary bodies. As the greatest numbers seemed to occur in the $30 \%$ adjunct zone, we included this range in an analysis of EIA reactive samples found during routine testing.

\section{Methods}

Endocervical and urethral swabs from women, or urethral swabs from men attending the Sexually Transmitted Diseases (STD) Clinic, Family Planning Clinic and Sexual Health Clinics, Calgary, were evaluated for this study. When possible, a paired set of urethral and cervical swabs was obtained from female patients attending the STD Clinic.

Patients from the STD Clinic were considered to have a higher prevalence (high risk group) than the other clinics (medium risk group) and the data were analysed by each specimen's clinic of origin.

The Syva MicroTrak EIA (Syva, Palo Alto, California, USA) was used to screen the samples and the assay was performed as stipulated by the manufacturer. The positive threshold was determined according to kit protocol. An adjunct category included those samples with absorbance values in a range from $30 \%$ below to the positive threshold. All reactive samples were verified by a confirmatory assay as described later. In addition, samples with absorbance values in the adjunct range, and both urethral and cervical swabs of a paired set when one of these gave an absorbance in the ranges described previously, were also verified.

For confirmatory testing, specimen buffer $(400 \mu \mathrm{l})$ was centrifuged at $10000 \times g$ for 10 minutes in a microfuge. The deposit was washed once in phosphate buffered saline, and resuspended in $200 \mu \mathrm{l}$ of the same. Fifty microlitres were spotted on to a slide, air-dried and fixed in methanol, then stained by the Syva MicroTrak DFA reagent (Syva) as described by the manufacturer. The stained slides were examined at $\times 400$ or $\times 500$ for the presence 
Table 1 Comparison of ELA and DFA result combinations with specimen site for female patients with paired sets, where one sample is $E L A$ reactive and DFA positive

\begin{tabular}{llll}
\hline & & \multicolumn{2}{l}{ Numbers and site of each swab } \\
\cline { 3 - 4 } EAIGDFA results & No. of patients & Cervical & Urethral \\
\hline EIA (R) DFA (P) & $85^{*}$ & 86 & 86 \\
EIA (R) DFA (N) & 4 & 0 & 4 \\
EIA (NR) DFA (N) & $53^{*}$ & 44 & 10 \\
EIA (NR) DFA (P) & 4 & 1 & 3 \\
EIA (H) DFA (P) & 7 & 1 & 6 \\
EIA (H) DFA (N) & 5 & 0 & 5 \\
\hline
\end{tabular}

* Two separate paired sets received from one patient with the same result.

$\mathrm{R}=$ absorbance value $\geq$ kit reactive threshold; $\mathrm{H}=$ absorbance value in range from $30 \%$ below to reactive threshold; $\mathrm{NR}=$ absorbance value less $<30 \%$ below reactive threshold; $\mathbf{P}=$ positive ( $\geq 1$ elementary body present); $\mathrm{N}=$ negative (no elementary bodies present).

Table 2 Numbers (\%) of specimens in each quotient category, with confidence limits

\begin{tabular}{|c|c|c|c|c|c|}
\hline \multirow[b]{2}{*}{ Patient group } & \multirow[b]{2}{*}{ Total } & \multicolumn{4}{|c|}{ Quotient category } \\
\hline & & $\geq 0 \cdot 7-1 \cdot 0$ & $>1 \cdot 0-2 \cdot 0$ & $>2 \cdot 0-4 \cdot 0$ & $>4 \cdot 0$ \\
\hline \multicolumn{6}{|l|}{ High risk } \\
\hline EIA reactives & 607 & 94 & 108 & 132 & 273 \\
\hline $\begin{array}{l}\text { DFA confirmed } \\
(\%) \\
\text { confidence limit }\end{array}$ & $\begin{array}{l}495 \\
(81 \cdot 5)\end{array}$ & $\begin{array}{l}21 \\
(22 \cdot 3) \\
\text { ND }\end{array}$ & $\begin{array}{l}72 \\
(66 \cdot 7) \\
55\end{array}$ & $\begin{array}{l}129 \\
(97 \cdot 7) \\
94 \cdot 3\end{array}$ & $\begin{array}{l}273 \\
(100)\end{array}$ \\
\hline \multicolumn{6}{|l|}{ Medium risk } \\
\hline EIA reactives & 287 & 35 & 48 & 80 & 124 \\
\hline DFA confirmed & 258 & 22 & 33 & 79 & 124 \\
\hline (\%) & $(89.9)$ & $(62 \cdot 9)$ & $(68 \cdot 8)$ & $(98 \cdot 8)$ & $(100)$ \\
\hline confidence limit & & ND & $51 \cdot 6$ & $95 \cdot 7$ & 100 \\
\hline
\end{tabular}

of elementary bodies (EB) using an ultraviolet epifluorescence microscope. Although slides with $>5 \mathrm{~EB}$ were routinely regarded as positive, those slides with between 1 and 5 EBs required consensus between two experienced technologists to be considered positive. Positive and negative controls from the EIA kit were treated and stained as described above.

Standard statistical formulae ${ }^{67}$ for sensitivity, specificity and confidence limits were applied where stated.

\section{Results}

A total of 13283 swabs from 9583 patients from both risk groups were evaluated. In the high risk group there were 7646 specimens from 4551 patients distributed by sex and site as follows: 3153 male urethral swabs, 2262 female urethral swabs, and 2231 cervical swabs. In the medium risk group 5637 endocervical swabs from 5032 female patients were tested; no urethral swabs were collected. A total of $765(5 \cdot 8 \%)$ were EIA reactive, 513 of 7646 $(6.7 \%)$ from the high risk group and 252 of $5637(4.5 \%)$ from the medium risk group.

After DFA testing, 474 of $513(92 \cdot 4 \%)$ swabs from 374 high risk group patients, 161 women and 213 men, were confirmed as positive. The distribution and percentages for these positive swabs by site were as follows: $224(7 \cdot 1 \%)$ male urethral swabs, $101(4.5 \%)$ female urethral swabs, and $149(6 \cdot 7 \%)$ cervical swabs. A comparison of confirmed positive versus EIA reactive results by site was as follows: 149 positive results from $157(94.9 \%)$ reactive cervical swabs, 101 from $113(89.4 \%)$ female urethral swabs, and 224 from $243(92 \cdot 2 \%)$ male urethral swabs. No statistical difference was found between sites for DFA negative, EIA reactive samples.
When those samples with absorbance values within the adjunct range were included, an additional 94 swabs from the high risk group were also tested by DFA (20 cervical swabs, 30 female urethral swabs, and 44 male urethral samples). After DFA testing, 21 of 94 (22.3\%) swabs from 21 patients also contained elementary bodies (12 male urethral, five female urethral, and four cervical swabs). These 21 additional patients, nine women and 12 men, represented $5.6 \%$ ( 21 of 374 ) of the total number of patients with DFA confirmed specimens. The nine women with sample absorbance values in the $30 \%$ adjunct range all had paired sets of samples collected. For two women, the alternate swab of the pair was negative on both EIA and DFA, whereas for the remaining seven, the alternate swab of the pair was EIA reactive and DFA positive.

Table 1 shows the results of paired urethral and cervical swabs from 158 women from the high risk group when one of the pair of samples taken was EIA reactive and DFA positive. In $53 \%$ of these female patients both swabs were EIA reactive and DFA positive, and $33 \%$ had only one of the pair as a confirmed reactive. A number of other combinations were found and comprised about $13 \%$ of the total. There were four women from whom one swab was EIA reactive but DFA negative.

In the medium risk group $93.7 \%$ (236 of 252) of EIA reactive samples were confirmed as true positives (collected from 236 patients). Within the $30 \%$ adjunct range, 35 additional samples required verification and $22(62.9 \%)$ of these (from 22 women) were confirmed as positive by DFA. This represented $9.3 \%$ of the total number of patients with confirmed, reactive swabs ( 22 of 236 ) compared with $5.6 \%$ from the high risk group.

With the MicroTrak DFA as the reference, the sensitivity of the EIA test was determined to be 95.7 and $91.5 \%$ for the high and medium risk groups, respectively. Similarly, specificities of 92.3 and $93.6 \%$, respectively, were determined based on the kit criteria. Prevalences of 8.0 and $4.6 \%$ were calculated for the high and medium risk groups, respectively, based on the laboratory data (after correction for multiple swabs from individual patients).

An absorbance quotient for each DFA positive specimen was obtained by division of the specimen absorbance by the test specific cut off value and these results are shown in table 2 . In the high risk group, 273 of $513(53 \cdot 2 \%)$ EIA reactive samples gave a quotient of $\geq 4.0$ with respect to the cut off value, and all of these were confirmed as DFA positive. As the quotient decreased, the number of DFA confirmed samples declined. Twenty one samples were identified as DFA positive in the $\geq 0.7$ to 1.0 range. These would have been missed based on the kit criteria of an EIA reactive result. There were an additional five swabs all from paired sets, four urethral and one cervical swab, which had quotients in the range from 0.2 to 0.4 but which were DFA positive (data not shown). Similarly for the medium risk group, $124(49 \cdot 2 \%)$ specimens had quotients of $\geq 4 \cdot 0$ and all were DFA positive (table 2). Twenty 
two patients were identified as DFA positive when the quotients were in the range of $\geq 0.7$ to $1 \cdot 0$.

Thus, for both risk groups a combined total of 36 of 610 patients $(5 \cdot 9 \%)$ with DFA confirmed results (22 women from the medium risk group and 12 men and two women from the high risk group) would have been missed if the kit criteria for a reactive result were strictly applied.

A $99 \%$ confidence limit was applied to each quotient range for both risk groups (table 2). False positive results were likely despite relatively high $(>2.0$ to 4.0$)$ absorbance values. Lower limits were not calculated for the $\geq 0 \cdot 7$ to 1.0 category as this represented a range below the EIA reactive threshold.

\section{Discussion}

In this study the results from a large number of specimens revealed the range of confirmed and unconfirmed EIA reactive samples that would be encountered in a diagnostic setting. Analysis by specimen origin and site showed that the sensitivity and specificity were similar between the two risk groups of varying disease prevalence, indicating that the kit performed equally in both. Furthermore, the lack of a statistical difference between the DFA confirmed and unconfirmed specimens provided additional support. Similar sensitivities have been found in other studies. ${ }^{910}$ Although we noted a lower specificity in our study compared with another, ${ }^{9}$ this may be explained by the DFA being performed on all our reactive specimens rather than on selected samples.

A number of studies ${ }^{1-3}$ have applied the DFA to verify EIA reactive results from alternative kits to the one we used. Schwebke et $a l^{3}$ noted that the specificity could be improved to almost $100 \%$ by verifying swabs with absorbance quotients over a wide range both above and below the positive threshold. A consequence of this wide quotient range was that at least $25 \%$ of the samples required testing by DFA compared with $14 \%$ in our study, of which one third were confirmed as positive. In the other studies, ${ }^{12}$ DFA testing was only performed when discrepancies were observed between the various assays.

From other studies, ${ }^{11} 12$ where an alternative EIA kit to the one here was used, it was noted that a proportion of specimens with reactive absorbance values were not confirmed on DFA testing, and that this proportion declined as the absorbance values increased. We applied a stringent $99 \%$ confidence level to assess the reliability of the EIA results for the different quotient categories. From our results, an average of about $50 \%$ of EIA reactive tests from both groups could not be equivocally relied upon until this quotient exceeded $4 \cdot 0$. Thus, specimens with absorbance values equivalent to a quotient of less than this value should be verified by an alternative procedure.

The problem of false negative results has been recognised with all EIA kits. ${ }^{135910}$ By incorporating an adjunct $30 \%$ range below the manufacturer's positive cut off value, we were able to extend the sensitivity of this assay and thereby confirm an additional 22 patients from the medium risk group and 14 from the high risk group as positive for $C$ trachomatis, who would otherwise have been missed if the kit protocol had been strictly adhered to. The DFA verification results of these apparently EIA nonreactive samples showed that the numbers of elementary bodies were mostly greater than five per slide, although not all confirmations were routinely quantified (data not shown). The lack of correlation between numbers of elementary bodies and absorbance values in this adjunct range has been published. ${ }^{8}$ It is likely that there were other DFA positive samples which would have screened as non-reactive, but as it was impractical to verify all specimens by the DFA, these numbers are unknown.

Detection of true positive results among specimens with absorbances in the adjunct range is a potential benefit of this scheme, although it does require extra technical time in order to verify the additional samples, many of which are likely to be negative. However, the numbers in our study were relatively low (129 swabs) in relation to the total numbers of specimens tested: this justifies the time spent on the verification procedure.

As with the EIA, the DFA verification test is also prone to false negative results. In our study there were four of $89(4.5 \%)$ samples from paired sets of cervical and urethral swabs when one was both EIA reactive and DFA positive and the other swab although EIA reactive, was not confirmed by the DFA. By applying this percentage to those reactive specimens unconfirmed by DFA it is likely that an additional three patients from both risk groups would have been reported as falsely negative.

In summary, we accept that the screening test will not identify all positive samples, although the $30 \%$ adjunct zone provides a compromise which detects additional patients without verification of an unduly large number of specimens. Furthermore, although all EIA reactive samples above the recommended cut off value should be verified in order to identify potential false positive results, from a practical standpoint it would be appropriate to confirm only those with a quotient of between 1 and 4 with respect to the positive cut off value.

The authors acknowledge the contribution and assistance of the staff of the Virology Department, Provincial Laboratory, Calgary, Canada, the Calgary Sexually Transmitted Disease Clinic (Alberta Health) and the Calgary Family Planning Clinic \& Sexual Health Clinic (Calgary Health Services).

1 Moncada J, Schachter J, Bolan G, Engelman J, Howard L, Mushahwar I, et al. Confirmatory assay increases specificity of the Chlamydiazyme test for Chlamydia trachomatis infection of the cervix. F Clin Microbiol 1990;28 1770-3.

2 Olsen MA, Sambol AR. Comfirmation of positive results for Chlamydial antigen by the Chlamydiazyme assay: value of repeated testing and a blocking antibody assay. 7 Clin Microbiol 1993;31:1892-5.

3 Schwebke JR, Stamm WE, Hunter Handsfield H. Use of sequential enzyme immunoassay and direct fluorescen antibody tests for detection of Chlamy infections in women. 7 Clin Microbiol 1990;28:2473-6.

4 LeBar W, Schubiner H, Jemal C, Herschman B, Criswell $\mathrm{K}$. Curtin N, et al. Comparison of the Kallested Pathfinder K, Curtin N, et al. Comparison of the Kallested Pathfinder ELA, cytocentrifuged direct fluorescent antibody, and cell culture for the detection of Chlamydia trachomatis. Diagn

5 Clark A, Stamm WE, Gaydos C, Welsh L, Quinn TC, 
Schachter J, et al. Multicentre evaluation of the AntigEnz Chlamydia enzyme immunoassay for diagnosis of Chlamydia trachomatis genital infection. $f$ Clin Microbio 1992;30:2762-4.

6 Bland M. An introduction to medical statistics. Oxford: Oxford Medical Publications, 1989.

7 Ilstrup DM. Statistical methods in microbiology. Clin Microbiol Rev 1990;3:219-26.

8 Beebe JL, Rau MP, Albrecht KD. Confirmatory testing of Chlamydia trachomatis Syva enzyme immunoassay gray zone specimens by Syva direct fluorescent antibody test. Sex Transm Dis 1993;20:140-2.

9 Gaydos CA, Reichart CA, Long JM, Welsh LE, Neumann TM, Hook EW III, et al. Evaluation of Syva enzyme immunoassay for detection of Chlamydia trachomatis in genital specimens. $\mathcal{F}$ Clin Microbiol 1990;28:1541-4.

10 Moncada J, Schachter J, Bolan G, Nathan J, Shafer MA, Clark A, et al. Evaluation of Syva's enzyme immunoassay for the detection of Chlamydia trachomatis in urogenital specimens. Diagn Microbiol Infect Dis 1992; 15:663-8.

11 Taylor-Robinson D, Thomas BJ, Osborn MF. Evaluation of enzyme immunoassay (Chlamydiazyme) for detecting Chlamydia trachomatis in genital tract specimens. $\mathcal{f}$ Clin Pathol 1987;40:194-9.

12 Chisholm SM, Matheson BA, Ho-Yen DO. Limitations of Chlamydiazyme in general hospital laboratories [letter]. $\mathcal{f}$ Clin Pathol 1988;41:357-8. 